\title{
PERCEPÇÕES DE GESTANTES SOBRE O PRÉ-NATAL ODONTOLÓGICO
}

\author{
PERCEPTIONS OF PREGNANT WOMEN ABOUT DENTAL PRENATAL CARE \\ PERCEPCIONES DE MUJERES EMBARAZADAS ACERCA DE LA ATENCIÓN PRENATAL \\ ODONTOLÓGICA
}

Lívia Karynne Martins Mesquita ${ }^{1}$, Ana Carolina Souza Torres ${ }^{2}$, José Osmar Vasconcelos Filho ${ }^{3}$

\begin{abstract}
RESUMO
Analisar as percepções de gestantes sobre o pré-natal odontológico em um município do Nordeste brasileiro. Pesquisa qualitativa, desenvolvida em Crateús, no Ceará, de junho de 2020 a fevereiro de 2021, na Unidade de Saúde da Família Maratoan. Os dados foram coletados por meio de entrevistas semiestruturadas, com a participação de 12 gestantes. O material empírico gerado foi transcrito e analisado pela técnica de análise de conteúdo do tipo temática. As categorias temáticas geradas foram: Autopercepções de gestantes acerca da importância do pré-natal odontológico; Fluxos de acesso ao pré-natal odontológico; Medo ou trauma de dentista. As gestantes participantes reconheceram a importância do pré-natal odontológico, mas com pouca clareza dos motivos. A assistência odontológica na gestação é permeada de mitos e desconfianças, e a falta de vínculo entre equipe de saúde e pacientes é um dos fatores que mais afastam as gestantes do dentista. A escuta qualificada do dentista no pré-natal odontológico pode desmistificar os possíveis medos e traumas das gestantes e incentivar a criação e a fortificação da relação profissional-paciente. Faz-se necessário investir na educação em saúde, tendo a gestante como protagonista do próprio cuidado, apoiada por práticas profissionais de cuidado desenvolvidas em grupos dinâmicos, interativos e multidisciplinares.
\end{abstract}

Palavras-Chave: Saúde Bucal; Cuidado Pré-Natal; Atenção Primária à Saúde.

\section{ABSTRACT}

This study aimed to analyze the perceptions of pregnant women about dental prenatal care in a city in northeastern Brazil. It was a qualitative research conducted in Crateús, Ceará, from June 2020 to February 2021, at the Maratoan Family Health Unit. Data collection occurred through semi-structured interviews with 12 pregnant women. The empirical material developed was transcribed and analyzed using the thematic content analysis technique. The following thematic categories emerged: Self-perception of pregnant women about the importance of dental prenatal care; Access flows to dental prenatal care; Dental phobia or trauma. Participants recognized the importance of prenatal dental care, but not so sure why. Dental care during pregnancy is permeated with myths and mistrust, and the lack of bonding between the health team and patients is one of the factors that most keep pregnant women away from the dentist. Qualified listening by the dentist in dental prenatal care can demystify possible fears and traumas of pregnant women and encourage the creation and strengthening of professional-patient relationship. There is need to invest in health education, with pregnant women as protagonists of their own care, supported by professional care practices developed in dynamic, interactive, and multidisciplinary groups.

Keywords: Oral Health; Prenatal Care; Primary Health Care.

\section{RESUMEN}

Analizar percepciones de gestantes sobre atención prenatal odontológica, en ciudad del noreste brasileño. Investigación cualitativa, desarrollada en Crateús, Ceará, de junio/2020 a febrero/2021, en la Unidad de Salud de la Familia Maratoan. Datos recolectados a través de entrevistas semiestructuradas, con 12 gestantes. Material empírico transcrito y analizado mediante técnica de análisis de contenido temático. Las categorías temáticas generadas fueron: Autopercepciones de gestantes sobre la importancia de la atención prenatal odontológica; Flujos de acceso a la atención prenatal odontológica; Miedo o trauma del dentista. Las participantes reconocieron la importancia de la atención prenatal odontológica, pero con poca claridad sobre sus razones. La atención odontológica durante el embarazo está impregnada de mitos y desconfianzas, y la falta de vínculo entre equipo de salud y pacientes es uno de los factores que más distancia a la embarazada del dentista. La escucha calificada del odontólogo en la atención prenatal odontológica puede desmitificar

\footnotetext{
${ }^{1}$ Escola de Saúde Pública do Ceará, Fortaleza, Brasil. (0000-0003-0579-1414)

2 Universidade Federal da Bahia, Salvador, Brasil. (0000-0002-8837-4781)

${ }^{3}$ Universidade Estadual de Campinas, São Paulo, Brasil. (0000-0002-4313-0484)
} 
posibles miedos y traumas de la gestante y favorecer la creación y fortalecimiento de la relación profesional-paciente. Es necesario invertir con educación en salud, tiendo la gestante como protagonista del propio cuidado, sustentada en prácticas de cuidado profesional desarrolladas en grupos dinámicos, interactivos y multidisciplinarios.

Palabras Clave: Salud Bucal; Atención Prenatal; Atención Primaria de Salud.

\section{INTRODUÇÃO}

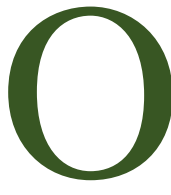

pré-natal odontológico é uma demanda real e necessária já implementada nas Unidades Básicas de Saúde (UBS), referenciada por diversas políticas, entre elas a Rede Cegonha, que tem como objetivo estruturar a atenção à saúde materno-infantil no Brasil. Essa insere o Cirurgião-Dentista (CD) no atendimento básico à gestante, como profissional necessário para o acompanhamento integral ${ }^{1}$.

Devido às mudanças que ocorrem em gestantes nos âmbitos psicológico, físico $\mathrm{e}$ hormonais, estas passam a constituir um grupo de pacientes de temporário risco odontológico. Somase a isso o fato de o atendimento odontológico ser assunto de extrema controvérsia, principalmente em função das crenças sem comprovação científica, tanto por parte de gestantes, afastandoas do consultório odontológico, como também dos cirurgiões-dentistas que, muitas vezes, sentem-se incapacitados para atendê-las e realizar procedimentos de maneira segura ${ }^{2}$.

$\mathrm{O}$ atendimento odontológico às gestantes deve ser realizado em qualquer período gestacional, sendo o segundo trimestre $\mathrm{o}$ preferencial para realização de procedimentos invasivos. Nenhuma necessidade materna deve ser negligenciada. É importante para se conseguir suprir as demandas que existem na gestação entrosamento satisfatório entre os profissionais que fazem parte da unidade de Estratégia Saúde da Família (ESF) ${ }^{3}$.

Existem poucos estudos sobre a utilização dos serviços odontológicos entre mulheres grávidas no Brasil. Desses, quase todos se dedicam a conhecer o perfil e fatores associados à utilização do serviço durante o pré-natal, e pouco se sabe sobre as percepções das gestantes que estão frequentando os serviços de saúde, mas que não buscam o atendimento odontológico. E são exatamente estas que apresentam maior risco de estarem com problemas bucais, assim como os recém-nascidos no futuro ${ }^{4}$.

O presente estudo aborda temática que sinaliza a necessidade de desenvolver estratégias de educação em saúde bucal com gestantes. Dessa forma, a assistência odontológica no pré-natal se fortalecerá enquanto parte do tratamento odontológico que suscitará motivação e adoção de novos hábitos em pacientes, o que contribuirá com a promoção de saúde bucal do binômio mãe-filho, bem como com a desmistificação de crenças populares que menosprezam $\mathrm{o}$ atendimento odontológico durante a gestação.

A escolha e implicação com esse objeto de pesquisa surgiu a partir das vivências enquanto profissional de saúde - residente da Residência Integrada em Saúde, da Escola de Saúde Pública do Ceará (RIS-ESP/CE). Tal formação oferece oportunidade de atuação diferenciada aos profissionais da saúde, principalmente aos cirurgiões-dentistas, que historicamente foram os últimos a integrar a ESF, que foge à realidade da rotina da ESF: atendimentos compartilhados, participação ativa em grupos de promoção de saúde e atendimentos individuais.

Durante a rotina de atendimentos individuais e compartilhados, observou-se o desinteresse de gestantes em passar pelo acompanhamento odontológico. Tanto o desconhecimento sobre o atendimento ofertado pela odontologia durante o pré-natal como o medo atrelado a traumas passados e mitos justificam a recusa em aceitar o tratamento. A pouca informação ou a distorção desta, assim como o medo de ir ao dentista, podem constituir as principais causas de afastamento e resistência ao tratamento odontológico 5 . Diante do exposto, objetivou-se analisar as percepções de gestantes 
sobre o pré-natal odontológico em um município do Nordeste brasileiro.

\section{METODOLOGIA}

Pesquisa com abordagem qualitativa, desenvolvida de junho de 2020 a fevereiro de 2021, a qual teve como cenário a UBS Maratoan, na cidade de Crateús-CE, Brasil. A UBS Maratoan é constituída por uma equipe de Saúde da Família (composta por dois enfermeiros, um médico, dois técnicos de enfermagem, um cirurgião-dentista e um técnico de saúde bucal) e uma equipe de Saúde Indígena (composta por enfermeiro, odontólogo, médico, técnico de enfermagem, técnico de saúde bucal e assistente social). As práticas desempenhadas por esses profissionais de saúde são pautadas na multiprofissionalidade, colaboração interprofissional, educação permanente, promoção da saúde e integralidade do cuidado. A justificativa para escolha desse local de pesquisas se deve ao fato dos profissionais de saúde, residentes em Saúde da Família e Comunidade da Residência Integrada em Saúde da ESP-CE, terem como campo de prática de atuação esse território.

Os informantes da pesquisa foram selecionados conforme os critérios: mulheres que se encontravam gestantes em qualquer período gestacional e que buscaram o atendimento prénatal na Unidade de Saúde Maratoan, durante a realização do estudo. As gestantes assistidas na UBS pesquisada foram convidadas a participarem do estudo de forma presencial, por meio de convite verbal da pesquisadora responsável.

Em decorrência da pandemia por SARSCoV-2, muitas gestantes tiveram dificuldades de sair de casa para realizar atendimento na UBS Maratoan, mas todas que compareceram à UBS, no período da pesquisa, aceitaram participar do estudo, o qual contou com a adesão de doze gestantes.

As gestantes confirmaram o consentimento em participar da pesquisa com a assinatura do Termo de Consentimento Livre e Esclarecido
(TCLE). As informações foram construídas por meio de entrevistas individuais, com roteiro semiestruturado que teve como recurso de registro a gravação, em que se estabeleceu uma situação de conversa informal, guiada por temas. $\mathrm{O}$ ambiente era neutro e propiciou conforto às gestantes para exposição de opiniões.

Dividiu-se a estrutura do roteiro em dois campos: um para caracterização da gestante, que era composto por dados pessoais, socioeconômicos e sobre a gestação; e outro campo para analisar as percepções das gestantes acerca da importância da assistência odontológica durante o pré-natal.

$\mathrm{O}$ roteiro semiestruturado contemplou questionamentos alinhados com os objetivos do estudo, por meio dos questionamentos: Considera que a gestação prejudica a saúde bucal? Tem medo de ir ao dentista? Foi informada antes ou durante a gestação da importância de ir ao dentista durante esse período? Se foi informada, quem passou a informação? Tem ido às consultas agendadas com o dentista? Com que frequência? Quando não vai às consultas com o dentista, tem algum motivo específico? Considera perigoso ir ao dentista estando gestante? Considera que pode transmitir alguma doença bucal para o seu bebê? Considera perigoso realizar radiografia odontológica durante a gestação? Tem alguma experiência anterior com o dentista que foi traumática?

Após a transcrição das entrevistas, procedeu-se a análise de conteúdo do tipo temática do material empírico. Para tanto, realizou-se leitura aprofundada do material transcrito e selecionaramse os trechos com as estruturas mais relevantes e ideias centrais (núcleos de sentido), agrupando-os e classificando-os em categorias temáticas ${ }^{6}$.

A pesquisa seguiu as normatizações éticas instituídas pela Resolução $n^{\circ} 466 / 2012$ do Conselho Nacional de Saúde (CNS) e foi aprovada pelo Comitê de Ética em Pesquisa com Seres Humanos da Escola de Saúde Pública do Ceará, conforme parecer $\mathrm{n}^{\circ}$ 4.068.9007.

$\mathrm{Na}$ escrita dos resultados, as informantes foram representadas pelo código "Gestante", 
seguido de numeral, como forma de preservar as identidades.

\section{RESULTADOS}

As doze gestantes entrevistadas tinham idades entre 15 e 41 anos, sendo a idade média delas de 26,25 anos; 58,4\% das gestantes se consideraram pardas, $33,3 \%$ pretas e $8,3 \%$ brancas. Em relação ao estado civil, $41,6 \%$ estavam casadas, $33,4 \%$ encontravam-se em união estável e $25 \%$ estavam solteiras. Em relação à escolaridade, 41,6\% declararam ter concluído o Ensino Médio, $25 \%$ não concluíram o Ensino Médio completo, 16,8\% não concluíram o Ensino Superior, 8,3\% finalizaram o Ensino Superior e 8,3\% não finalizaram o Ensino Fundamental. Dentre elas, $66,6 \%$ reportaram ter trabalho remunerado. A renda familiar de $50 \%$ delas era de um salário mínimo, 41,6\% menos de um salário mínimo e 8,4\% de dois salários mínimos. Nenhuma era ou havia sido fumante. Em relação ao número de gestações, $58,3 \%$ estavam na primeira gestação, $25 \%$ na segunda gestação e $16,7 \%$ estavam na terceira e quarta gestação. A idade gestacional variou entre quatro e quarenta semanas.

A análise do material empírico gerou as categorias temáticas: Autopercepções de gestantes acerca da importância do pré-natal odontológico; Fluxos de acesso ao pré-natal odontológico; e Medo ou trauma de dentista.

A categoria Autopercepções de gestantes acerca do pré-natal odontológico revelou que a maioria das gestantes considerou importante a assistência odontológica durante o pré-natal, mesmo não conseguindo explicar claramente os motivos, como percebido nos relatos a seguir:

"[...] eu acho importante. Porque eu acho que não só o corpo da gente, mas a saúde bucal da gente também é importante nesse momento. Eu acho que qualquer problema que a gente tiver vai fazer mal à criança também... Importante porque é uma saúde toda, começa na boca e vai." (Gestante 3)
"[...] é importante para ver também como é que está, que devido a gente estar nesse período de gravidez, eu creio que a gente come bastante (fala entre risos), e tudo o que vê pela frente, não é igual antes da gestação, eu creio que tem que ter um certo cuidado a mais." (Gestante 5)

Ainda nessa categoria, algumas usuárias também consideraram perigoso ir ao dentista estando grávidas, bem como arriscado realizar radiografia odontológica, mesmo achando que seria importante ir ao dentista nesse período, conforme se percebe nas falas que seguem:

“[... eu considero pela anestesia, é
perigoso.” (Gestante 9)
"[...] o Raio X prejudica a saúde da gente
e com certeza prejudicaria a criança."
(Gestante 7)

Na categoria Fluxos de acesso ao pré-natal odontológico, observou-se que a grande maioria das gestantes estava fazendo o acompanhamento odontológico e tinha fácil acesso ao serviço e à informação, mas que a pandemia por SARS-CoV2 mudou essa realidade, dificultando acesso e continuidade do cuidado na UBS, como se percebe nos relatos:

"[...] eu fui até no dia que deu certo, ai quando começou a pandemia, pronto, aí, foram canceladas as consultas." (Gestante 1)

"[...] é uma vez por mês, mas por causa da pandemia, mês passado, eu não fui." (Gestante 4)

Na categoria Medo ou trauma de dentista, verificou-se que as percepções da maioria das gestantes estavam atreladas às informações presentes na cultura social, no senso comum, que as afastavam do consultório odontológico, como nos relatos:

"[...] porque têm muitas histórias ruins que as pessoas contam de pessoas grávidas que vão ao dentista e acontecem coisas horrorosas. Durante a gestação, eu tenho medo." (Gestante 4) 
"[...] sim, porque, às vezes, a gente vai ao dentista e, às vezes, pode prejudicar a criança, pode fazer até que o parto seja prematuro. Corre risco a vida da gente e da criança." (Gestante 7)

Algumas gestantes relacionaram o medo às experiências odontológicas na infância ou aos procedimentos dependentes de anestesia, como percebido nas falas:
“[...] Tinha (risos) não sei por que quando criança, mas depois que eu usei aparelho, o medo passou." (Gestante 3)
“[...] é uma dor, às vezes, que a pessoa sente na hora de uma anestesia, alguma coisa assim e, aí, causa um constrangimento." (Gestante 5)

\section{DISCUSSÃO}

A assistência odontológica à gestante é uma questão de saúde pública em decorrência das mudanças fisiológicas e psicossociais peculiares da gestação. Portanto, as gestantes integram grupo especial, com caráter prioritário à assistência odontológica no Sistema Único de Saúde (SUS), a qual está pautada nas recomendações estabelecidas por diretrizes do Ministério da Saúde, como parte integrante dos cuidados durante o pré-natal. ${ }^{8,9}$

A atenção adequada à gestante deve ser conduzida por equipe de saúde multiprofissional e interdisciplinar. Tais práticas possibilitam exercer um cuidado pautado na integralidade da atenção, que constitui um dos princípios do $\mathrm{SUS}^{10}$. A qualidade da assistência pré-natal está a cada dia merecendo maior importância, devido ao aumento dos índices de mortalidades materna e perinatal ${ }^{11}$. Com tudo isso, é importante conhecer as gestantes e buscar a melhor maneira de integração equipe e gestante. $\mathrm{O}$ grupo de convivência vem se apresentado como importante ferramenta, pois ajuda a agregar os conhecimentos com troca de experiências.

Algumas gestantes relataram insegurança em relação aos procedimentos odontológicos, bem como quanto à anestesia a ser utilizada. A solução anestésica considerada padrão ouro para o atendimento às gestantes, em consonância com a grande maioria dos autores, é a lidocaína a 2\% com epinefrina na concentração de 1:100.000. O vaso constritor na solução anestésica é de suma importância e não há contraindicação, tendo como vantagem a concentração local dos anestésicos, diminuindo a toxidade sistêmica ${ }^{12}$.

As tomadas radiográficas foram apontadas pela maioria das gestantes entrevistadas neste estudo como inapropriadas. Pesquisa aponta que durante a gestação não há motivo para adiar os exames radiográficos, caso sejam necessários ${ }^{13}$. Porém, alguns cuidados devem ser tomados, como o uso de filmes ultrarrápidos, radiografias digitais, proteção com o avental de chumbo e uso de colar tireoidiano, além de evitar a repetição de tomadas radiográficas e, caso o tratamento possa ser adiado, esperar pelo terceiro trimestre.

Experiências traumáticas anteriores $\mathrm{e}$ relatos de familiares e de pessoas próximas reforçam a ideia que não se pode haver atendimento odontológico durante o período gestacional, como relatado por algumas das gestantes entrevistadas, o que se mostrou um dos principais fatores restritivos para que não busquem o atendimento odontológico. O sentimento de medo do dentista, presente nas falas das usuárias entrevistadas, corrobora as percepções analisadas em estudo realizado com primigestas em seis UBS de um município de São Paulo, Brasil, o qual evidenciou nas gestantes o mesmo sentimento, em que a experiência do tratamento odontológico pode ser perigosa ou dolorosa, sendo também referida como medo do dentista, o que representa barreira na busca por cuidados de saúde ${ }^{14}$. Esse contexto é justificado em virtude do passado histórico da odontologia, ligado a uma prática relacionada ao medo, à dor e tortura, praticados por barbeiros e práticos de uma odontologia mutiladora ${ }^{15}$.

Tem-se discutido estratégias para vencer o medo e o trauma associados à consulta odontológica. Assim, sugere-se ao $\mathrm{CD}$ que seja bom ouvinte, demonstre empatia, tenha calma e inspire confiança no paciente ${ }^{16}$. Logo, importante 
estratégia para ganhar a confiança do paciente são consultas mais longas, que permitam explicar os procedimentos e ouvir os anseios do usuário ${ }^{17}$. A comunicação dentista-paciente se mostrou fator decisivo para o sucesso na consulta odontológica.

Outro ponto que merece ser esclarecido para as gestantes é que o tratamento odontológico não causa nenhum prejuízo à saúde sistêmica da gestante e/ou bebê, pelo contrário, a falta de cuidado bucal e o desenvolvimento de patologias crônicas expandem a toxicidade feto placentária, o que pode arremeter a um quadro inflamatório materno-fetal ${ }^{18}$. Mulheres com periodontite têm risco mais elevado, podendo ser de três a sete vezes a mais de nascimento prematuro do que em mulheres sem a doença ${ }^{19}$.

Ao perceber a importância do acompanhamento odontológico no período gestacional e notar a carência deste e de outros serviços básicos, o Governo Federal, a partir da Portaria Ministerial $\mathrm{n}^{\circ} 2.979^{20}$, de 12 de novembro de 2019, instituiu o Programa Previne Brasil, que faz parte do novo modelo de financiamento do SUS, colocando como um dos indicadores que precisam ser cumpridos para o repasse de verbas a proporção de gestantes com atendimento odontológico realizado. O intuito desse indicador é estimular o vínculo da equipe com as gestantes e a realização do pré-natal odontológico.

Faz-se necessária prática políticopedagógica que estimule as atividades que visam à promoção, proteção e recuperação da saúde, respeitando a diversidade de culturas e conhecimentos, valorizando os saberes populares, a ancestralidade, o incentivo à produção individual e coletiva de conhecimentos e a introdução destes no $\mathrm{SUS}^{21}$. A educação popular em saúde é uma estratégia que contribui para a reversão da ausência de conhecimento, esclarecimento de dúvidas das gestantes, desmistificação do medo, traumas e inseguranças trazidas por essas usuárias, pois é pautada na construção de processos de cuidado dialogados, humanizados, acolhedores da cultura e do saber popular, de forma horizontal e igualitária.
Também influencia a adequação e criação de vínculo, antes mesmo da consulta individualizada ${ }^{22}$.

Em relação ao acesso às consultas odontológicas, as gestantes relataram descontinuidade nos atendimentos, devido à pandemia pelo novo coronavírus (SARS-CoV-2). No decorrer da pandemia, muitos órgãos, como Ministério da Saúde, a Agência Nacional de Vigilância Sanitária (ANVISA) e o Conselho Federal de Odontologia, lançaram notas técnicas que adequaram e restringiram o atendimento odontológico apenas para urgências e emergências. Nesse contexto, o CD esteve diante de novas funções, além das que já desempenhava anteriormente, pois também fez parte das equipes de triagem da Covid-19, escuta inicial e monitoramento domiciliar ${ }^{23}$.

Mesmo diante da magnitude da pandemia por SARS-CoV-2, não deve haver interrupção tão abrupta das ações de saúde, pois a população continua carente desses serviços e adoecendo. Com a intenção de prestar esse serviço de maneira remota, descreveram a nova tendência de continuidade do serviço como "teleodontologia", diante das restrições nos atendimentos presenciais, a qual mostrou-se como alternativa que oferece suporte e motivação à higiene oral e orientações gerais sobre possíveis patologias bucais ${ }^{24}$. Porém, mesmo diante de toda tecnologia do século XXI, deve-se pensar em quem não tem acesso à internet, celulares, computadores e na realidade de pessoas analfabetas. Assim, cogita-se: como vencer essa barreira de iniquidades sociais e conseguir levar saúde a toda população?

\section{CONSIDERAÇÕES FINAIS}

A realização do estudo permitiu o esclarecimento de dúvidas trazidas pelas usuárias participantes sobre o período gestacional, o que contribuiu para desmistificar medos e crenças, culturalmente construídas, a fim de evitar complicações na saúde em geral e bucal no período da gestação. A pesquisa favoreceu $o$ 
empoderamento das usuárias acerca do autocuidado na gestação, de modo a proporcionar benefícios que se estendem não somente para mães e filhos, como também para todo o ambiente familiar.

Como limitação, o desenvolvimento da pesquisa apontou para singularidades das gestantes pertencentes à realidade de um único cenário, fazendo-se necessário ampliar a investigação da temática, por meio de novos estudos que possam abarcar outras realidades da assistência odontológica às gestantes na Atenção Primária à Saúde (APS).

Conclui-se que o atendimento odontológico no período gestacional é repleto de mitos e desconfianças e que a carência de vínculo entre equipe de saúde e gestantes é um dos fatores que mais afastam as gestantes do dentista. A principal alternativa para mudar esse quadro seria investir na

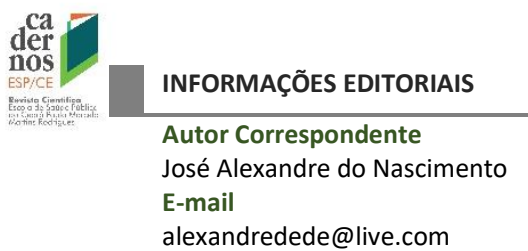

alexandredede@live.com educação em saúde, tendo a gestante como protagonista do próprio cuidado, sendo apoiada por práticas profissionais de cuidado desenvolvidas em grupos dinâmicos, interativos e multidisciplinares. O dentista também deve dedicar-se a um atendimento com escuta qualificada, no intuito de desmistificar possíveis medos e traumas e incentivar a criação e a fortificação da relação dentista-gestante.

\section{REFERÊNCIAS}

1. Pittner M, Bonassina M, Pittner E. Educação para a saúde bucal infantil: da gestação à idade pré-escolar. Rev UNINGÁ. 2016;27(2):22-9.

2 Matsubara AS, Demetrio ATW. Atendimento odontológico às gestantes: revisão da literatura. Rev UNINGÁ. 2017;29(2):42-7.

3 Silveira JLGC, Abraham MW, Fernandes CH. Gestação e saúde bucal: significado do cuidado em saúde bucal por gestantes não aderentes ao tratamento. Rev APS. 2016;19(4):568-74.

4 Konzen Júnior DJK, Marmitt LP, Cesar JA. Não realização de consulta odontológica entre gestantes no extremo sul do Brasil: um estudo de base populacional. Ciênc Saúde Colet. 2019;24(10):3889-96. doi: 10.1590/1413-812320182410.31192017.

5 Nunes Neto RA, Frutuoso MFP. Oral health and the care of pregnant women: workshops as a strategy to problematize practices in basic healthcare in residents living in the peripheral areas of the hills in the city of. Rev Gaúch Odontol. 2018;66(4): 305-16. doi: 10.1590/1981-863720180004000033504.

6 Minayo MCS. O desafio do conhecimento: pesquisa qualitativa em saúde. São Paulo: Hucitec; 2015.

7 Ministério da Saúde (BR), Conselho Nacional de Saúde. Resolução n ${ }^{\circ}$ 466, de 12 de dezembro de 2012. Aprova as diretrizes e normas regulamentadoras de pesquisas envolvendo seres humanos. Diário Oficial da União [Internet]. Brasília, 2013 jun 13 [citado em: 2021 Jul 23]; Seção1:59. Disponível em: https://conselho.saude.gov.br/resolucoes/2012/Reso466.pdf.

8 Ministério da Saúde (BR), Secretaria de Atenção à Saúde, Departamento de Atenção Básica, Coordenação Nacional de Saúde Bucal. Diretrizes da Política Nacional de Saúde Bucal [Internet]. Brasília; 2004 [citado em: 2021 Jul 23]. Disponível em: https://bvsms.saude.gov.br/bvs/publicacoes/politica_nacional_brasil_sorridente.htm. 
9 Botelho DLL, Lima VGA, Barros MMAF, Almeida JRA. Odontologia e gestação: a importância do pré-natal odontológico. SANARE. 2019;18(2).

10 Franco RVAB, Abreu LDP, Alencar OM, Moreira FJF. Pré-natal realizado por equipe multiprofissional da atenção primária à saúde. Cadernos ESP [online]. 2020 [citado 2021 Jul. 14];14(1):63-70. Disponível em: https://cadernos.esp.ce.gov.br/index.php/cadernos/article/view/247.

11 Andrade UV, Santos JB, Duarte C. A percepção da gestante sobre a qualidade do atendimento pré-natal em UBS, Campo Grande, MS. Rev Psicol Saúde. 2019;11(1):53-61.

12 Fabris V, Scortegagna AR, Oliveira GR, Scortegagna GT, Malmann F. Conhecimento dos cirurgiões dentistas sobre o uso de anestésicos locais em pacientes: diabéticos, hipertensos, cardiopatas, gestantes e com hipertireoidismo. J Oral Investig. 2018;7(1):33-51. doi: 10.18256/2238-510X.2018.v7i1.2468.

13 Miranda EB. Manejo odontológico em pacientes portadoras de diabetes gestacional [trabalho de conclusão de curso]. Gama (DF): Centro Universitário do Planalto Central Apparecido dos Santos; 2019. $6 \mathrm{f}$.

14 Fumagalli IHT, Lago LPM, Mestriner SF, Bulgarelli AF, Mestriner Júnior W. Percepções e atitudes de primigestas em relação à atenção em saúde bucal materno-infantil: um estudo qualitativo. Robrac: Rev Odontol Bras Central. 2021;30(89):44-63. doi: 10.36065/robrac.v30i89.1463.

15 Peronio TN, Silva AH, Dias SM. O medo frente ao tratamento odontológico no contexto do sistema único de saúde: uma revisão de literatura integrativa. Periodontia. 2019;29(1):37-43.

16 Batista TRM, Vasconcelos LMR, Vasconcelos MG, Vasconcelos RG. Medo e ansiedade no tratamento odontológico: um panorama atual sobre a versão na odontologia. Rev Salusvita. 2018;37(2):449-69.

17 Lemos PGS, Duque MAM, Machado CN. Componentes que afetam o medo no tratamento dentário em adultos: um estudo seccional. Braz J Implantol Health Sci. 2019;1(4):41-54.

18 Silva PNS, Deliberador TM, Gabardo MCL, Baratto-Filho F, Pizzatto E. Associação entre doença periodontal, parto prematuro e baixo peso ao nascer. Rev Cuba Estomatol. 2018;55(1):26-33.

19 Delgado JA, Santos PO, Alves MIM. A relação da doença periodontal com o parto prematuro. Rev Virtual ACBO [online]. 2019 [citado em: 2021 Jul. 23]; 8(1):20-4. Disponível em: http://www.rvacbo.com.br/ojs/index.php/ojs/article/view/399.

20 Ministério da Saúde (BR). Portaria no 2.979, de 12 de novembro de 2019. Institui o Programa Previne Brasil, que estabelece novo modelo de financiamento de custeio da Atenção Primária à Saúde [...]. Diário Oficial da União [Internet]. Brasília, DF; 2019 nov 13 [citado em: 2021 Jul. 23]; 220 (seção 1):97. Disponível em: https://www.in.gov.br/en/web/dou/-/portaria-n-2.979de-12-de-novembro-de-2019-227652180.

21 Fagundes DQ, Oliveira AE. Educação em saúde no pré-natal a partir do referencial teórico de Paulo Freire. Trab Educ Saúde. 2017;15(1):223-43. doi: 10.1590/1981-7746-sol00047.

22 Leal NAC, et al. Educação popular em saúde: desmistificando o pré-natal odontológico em um grupo de práticas corporais. Braz J of Dev [online]. 2021 [citado 2021 Jul. 23];7(4):37450-8. Disponível em: https://www.brazilianjournals.com/index.php/BRJD/article/view/28056.

23 Carletto AF, Santos FF. A atuação do dentista de família na pandemia do Covid-19: o cenário do Rio de Janeiro. Physis. 2020;30(3):1-10. doi: 10.1590/S0103-73312020300310.

24 Moura JFS, Moura KS, Pereira RS, Marinho RRB. COVID-19: a odontologia frente à pandemia. Braz J Health Rev . 2020;3(4):7276-85. 